EUROPEAN ORGANIZATION FOR NUCLEAR RESEARCH

Laboratory for Particle Physics

Divisional Report

CERN LHC/2001-7

(ECR)

\title{
CONCLUSIONS FROM 12 YEARS OPERATIONAL EXPERIENCE OF THE CRYOPLANTS FOR THE SUPERCONDUCTING MAGNETS OF THE LEP EXPERIMENTS
}

\author{
K. Barth, J.-P. Dauvergne, D. Delikaris and G. Passardi
}

\begin{abstract}
The Large Electron Positron Collider (LEP) has ended its last physics run in November 2000, and it is at present being dismantled to liberate the tunnel for the Large Hadron Collider (LHC) project to be completed by end of 2005. The cryogenic systems for the superconducting solenoid and focusing quadrupoles for the two LEP experiments, ALEPH and DELPHI, each supplying a cooling power of $800 \mathrm{~W} / 4.5 \mathrm{~K}$ entropy equivalent, have accumulated more then 100'000 hours of running time. The paper summarises the 12 years cryogenic experience in the various operating modes: cool-down, steady state, recovery after energy fast dump, utilities failures and warm-up of the superconducting magnets. The detailed operation statistics is presented and compared to the other CERN cryogenic systems. Emphasis is given to the technical analysis of the fault conditions and of their consequences on the helium refrigeration production time in view of the future operation of the LHC cryogenics.
\end{abstract}

LHC Division

Presented at the 2001 Cryogenic Engineering Conference and International Cryogenic Materials Conference CEC/ICMC 2001

16-20 July 2001, Madison, Wisconsin, USA

Administrative Secretariat LHC Division CERN

CH - 1211 Geneva 23
Geneva, Switzerland

30 October 2001 


\title{
CONCLUSIONS FROM 12 YEARS OPERATIONAL EXPERIENCE OF THE CRYOPLANTS FOR THE SUPERCONDUCTING MAGNETS OF THE LEP EXPERIMENTS
}

\author{
K. Barth, J.-P. Dauvergne, D. Delikaris, G. Passardi \\ CERN - European Organization for Nuclear Research \\ 1211 Geneva, Switzerland
}

\begin{abstract}
The Large Electron Positron Collider (LEP) has ended its last physics run in November 2000, and it is at present being dismantled to liberate the tunnel for the Large Hadron Collider (LHC) project to be completed by end of 2005. The cryogenic systems for the superconducting solenoid and focusing quadrupoles for the two LEP experiments, ALEPH and DELPHI, each supplying a cooling power of $800 \mathrm{~W} / 4.5 \mathrm{~K}$ entropy equivalent, have accumulated more then 100 '000 hours of running time. The paper summarises the 12 years cryogenic experience in the various operating modes: cool-down, steady state, recovery after energy fast dump, utilities failures and warm-up of the superconducting magnets. The detailed operation statistics is presented and compared to the other CERN cryogenic systems. Emphasis is given to the technical analysis of the fault conditions and of their consequences on the helium refrigeration production time in view of the future operation of the LHC cryogenics.
\end{abstract}

\section{INTRODUCTION}

CERN uses 21 liquid helium cryoplants for cooling a large variety of superconducting devices namely: accelerating cavities, magnets for accelerators and particle detectors. Six refrigerators ( $4 \times 18 \mathrm{~kW}$ at $4.5 \mathrm{~K}$ and $2 \times 400$ Watt at $4.5 \mathrm{~K}$ entropy equivalent) were used to cool the superconducting cavities and magnets of the Large Electron Positron collider (LEP) and Super Proton Synchrotron (SPS). The other 15 refrigerators supply cooling for superconducting magnets of various experiments as well as for cryogenic test facilities of the new Large Hadron Collider (LHC). The helium cryogenics is used by two different type of physics experiments at CERN:

The fixed-target experiments installed in the areas where the various SPS particle beams are extracted.

The experiments installed in the LEP e+e- collider. Two of the four detectors (ALEPH and DELPHI) used a superconducting solenoid integrated into the detector set-up and acting as a momentum analyser for the secondary particles produced in the collisions. All four experiments (L3, ALEPH, OPAL, DELPHI) had superconducting quadrupoles (low-b), strongly focussing the circulating beams on each side of the collision point, and thus increasing the beam luminosity for the experiment. 
TABLE 1. Design characteristics of the superconducting magnets in a LEP experimental area [1]

\begin{tabular}{l|cc}
\hline \multicolumn{1}{c}{ Magnet type } & $\begin{array}{c}\text { Detector solenoid } \\
(\text { ALEPH / DELPHI })\end{array}$ & Low-beta quadrupole \\
\hline Outer diameter (m) & $6.0 / 6.2$ & 0.48 \\
Warm bore diameter (m) & $5.0 / 5.2$ & 0.13 \\
Overall length (m) & $7.0 / 7.4$ & 2.5 \\
Nominal field or gradient & $1.5 / 1.2 \mathrm{~T}$ & $36 \mathrm{~T} \mathrm{~m}^{-1}$ \\
Nominal current (kA) & 5.0 & 1.63 \\
Stored energy (MJ) & $137 / 108$ & 0.33 \\
Cold mass (t) & 25 & 1.05 \\
Cooling method & Circulation of two-phase He & Liquid He bath \\
Liquid He capacity (l) & using thermo-siphon/ liquid pump & 50 \\
\hline
\end{tabular}

The main design characteristics of the superconducting magnets of the LEP experiments are given in TABLE 1 . In the following, a final summary of over 12 years of operational experience is given.

\section{CONSTRAINTS AND LAYOUT OF THE LEP EXPERIMENT CRYOGENICS}

The cryogenic system had to accommodate the strong differences between the two types of magnets regarding their dimensions and operation parameters (TABLE 1).

The cryogenic system had to provide the following refrigeration and liquefaction duties in fully automatic operation, supervised from a central control room and minimum local interventions for preventive or corrective maintenance (three month per year):

- Normal duty:

$2500 \mathrm{~W}$ refrigeration at a temperature below $75 \mathrm{~K}$ to the thermal shield of the detector solenoid. A mix of liquefaction and refrigeration at $4.5 \mathrm{~K}$ in varying proportions, depending upon the operation modes of the user magnets:

Solenoid (ramping / steady operation): $200 \mathrm{~W}+1.25 \mathrm{~g} / \mathrm{s} / 100 \mathrm{~W}+1.25 \mathrm{~g} / \mathrm{s}$

Quadrupole (cooldown / steady operation): $1.5 \mathrm{~g} / \mathrm{s} / 0.7 \mathrm{~g} / \mathrm{s}$

- $\quad$ Forced-flow cooldown:

To cool down the magnets from ambient temperature, the cryogenic system had to provide gaseous helium at a pressure of $1.2 \mathrm{MPa}$ (12 bar), at progressively decreasing temperatures with flow rates of $40 \mathrm{~g} \mathrm{~s}^{-1}$ for the solenoid and $5 \mathrm{~g} \mathrm{~s}^{-1}$ for the quadrupoles. Due to the different operation schedules the cooldown of the quadrupoles had to be performed without affecting the established steady operation of the detector solenoid.

- $\quad$ Recovery of rapid thermal transients:

Thermal transients provoked by current ramping and slow discharge in the superconducting magnets, as well as a fast dump, in case the of an emergency of the electromagnetic energy stored in the magnets, the re-establishment of steady operation conditions of the detector solenoid and the quadrupoles had to be done in parallel, sharing the maximal available refrigeration power between them.

The LEP experiments cryogenic system was divided into two main parts (see FIG 1): On the surface the gaseous helium storage, compression and purification system were installed, while the cold box, liquid helium storage and distribution system were located in the underground experiment caverns. The main technical characteristics of the cryogenic equipment are shown in TABLE 2, while further details can be found elsewhere [1-3]. 


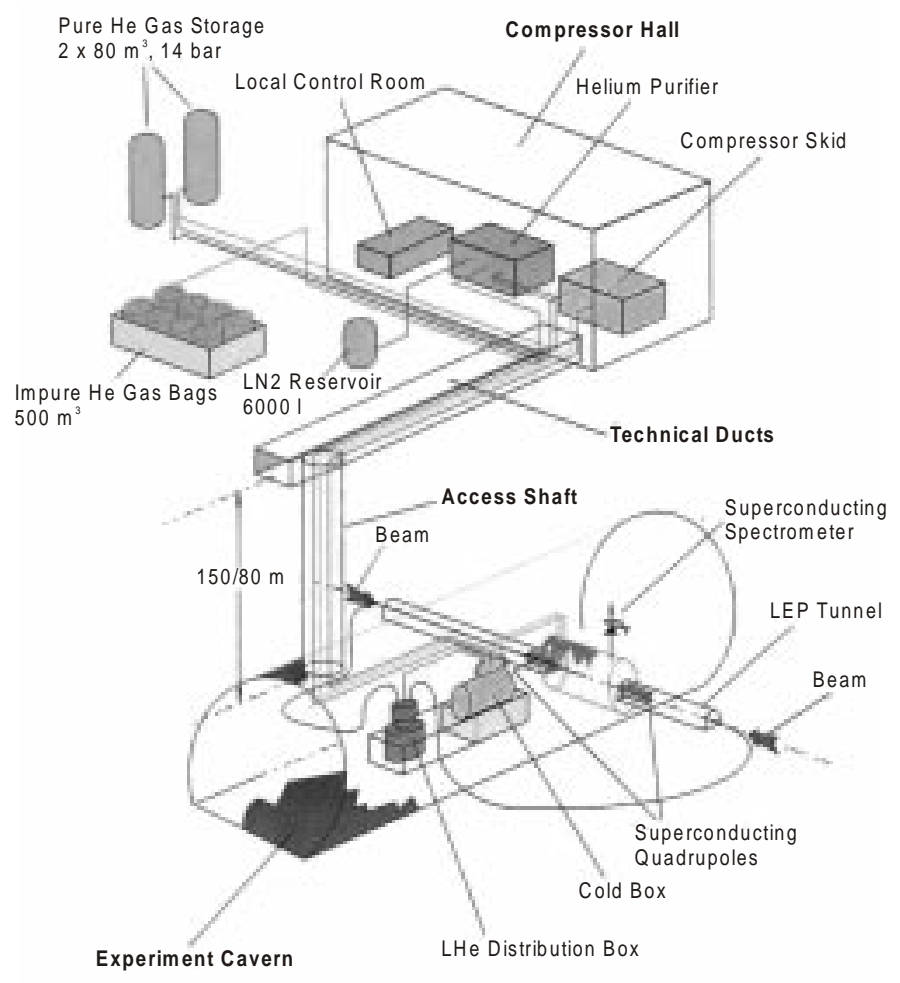

FIGURE 1. Schematic overview of a cryoplant for a LEP experimental site

\section{OPERATIONAL PERFORMANCE, STATISTICS AND FAULT ANALYSIS}

During the lifetime of the LEP accelerator the cryogenic systems for the experiments ALEPH and DELPHI accumulated more then 100,000 hours of running time. The major performances in the various operation modes over this very long operational time are analysed.

TABLE 2. Main characteristics of cryogenic equipment at a LEP experimental site [1]

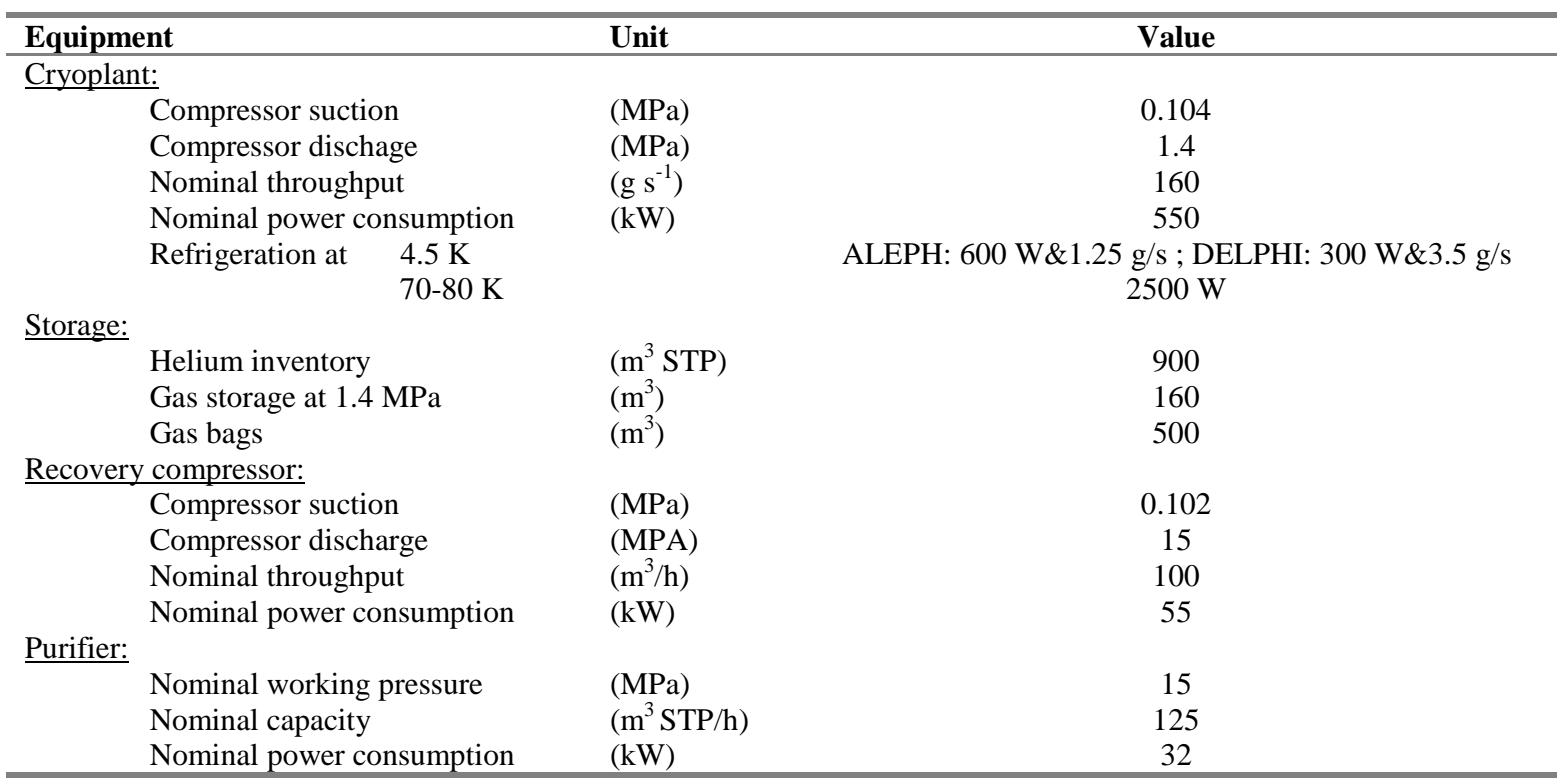




\section{Cooldown}

A cooldown from ambient temperature to steady operating conditions had to be done once per year, after a general maintenance shutdown lasting typically three months. FIG 2 shows the temperature variation for a typical controlled automatic sequential cooldown of the detector solenoid and low-beta quadrupoles, which took $180 \mathrm{~h}$ and $50 \mathrm{~h}$, respectively. During the cooldown the temperature gradients developed in the structures were kept low by limiting the maximum temperature difference between the cold gas and the magnets to less then $40 \mathrm{~K}$.

\section{Recovery from perturbations during cryogenic system operation}

The main causes for the occurring of thermal transients, which perturbed normal system operation, were:

- Loss of external utilities (electricity, cooling water, compressed air) required for cryoplant and magnet operation

- Failure of cryoplant equipment (valves, turbines, plant instrumentation etc.)

- Leakage of impurities (air, humidity) into the cryoplant cold helium circuits, provoking their partial or complete blockage and, as a consequence, the loss of refrigeration/liquefaction performance

- Faults in the detection of resistive transitions (quench) of the superconducting solenoids and quenches of quadrupoles induced by uncontrolled LEP beam losses

The cryogenic system was specially designed to handle all the above perturbations and to serve the two kinds of magnets with little interference. During a slow discharge triggered by a cryoplant stop, a buffer liquid storage for the detector solenoids (500 1 for ALEPH, 8001 for DELPHI) allowed maintaining the nominal liquid helium cooling, avoiding a warm-up of the $25 \mathrm{t}$ cold-mass. The $500 \mathrm{l}$ liquid-helium buffer-dewar for the superconducting quadrupoles allowed even autonomous operation for up to 60 min after a liquid helium production stop. Active control of the pressure in the gaseous helium return manifold, backed by self-actuated relief valves, maintained a constant back-pressure and therefore eliminated any interference between solenoid and quadrupole operation. This proved to be vital in the case of recovery from a slow or fast discharge of the detector solenoid, while keeping at the same time the quadrupole magnets in steady operation for continuing LEP accelerator running.
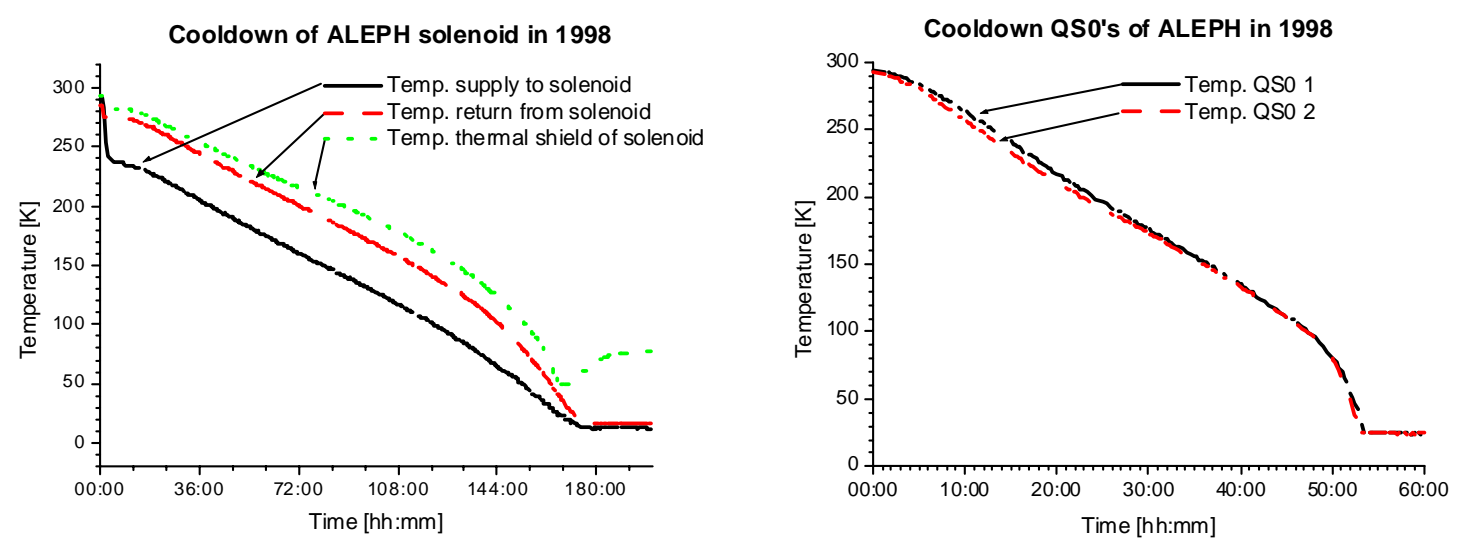

FIGURE 2. Temperature evolution for a typical cooldown of detector solenoid (left) and quadrupoles (right) 


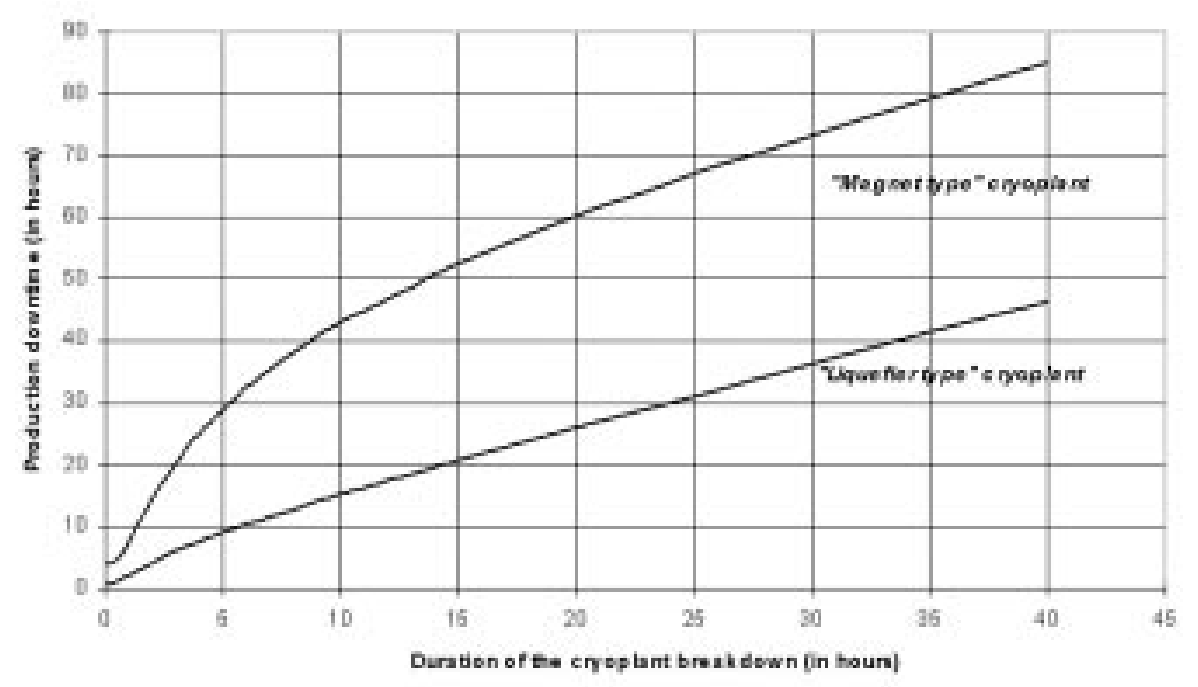

FIGURE 3. Typical recovery time profiles for "magnet type" and "pure liquefier type" mode of operation of CERN cryoplants

The graph shown in FIG 3 summarises the experience, acquired to a great extent with the ALEPH and DELPHI cryogenic systems, in recovering from a cryoplant stop. The total time required to re-establish nominal conditions is a factor of more than 4 times the cryoplant breakdown and repair time, between 0 and 10 hours. Afterwards the relation becomes linear and the amplification factor decreases to around 2. For comparison the same relation is shown for a liquefier mode of operation, which is less important but also emphasises the need of minimising the time needed to re-start the cryoplant.

The overcapacity of the cryogenic system proved to be particularly helpful for the recovery of process helium and re-establishment of steady operation conditions in the case of a fast discharge of the detector solenoid or the quadrupole magnets. Due to the limited liquid helium buffer the cooling circuits of the solenoid coil could not be maintained during a fast dump of the stored energy and the cold mass warmed up to around $40-60 \mathrm{~K}$. Nominal operation conditions could be restored automatically after such a quench within 12 - 18 hours (left graph in FIG 4) and without disturbing the superconducting quadrupole operation. Automatic quench recovery of the superconducting quadrupoles could be accomplished within 50-60 min., due to the smaller cold mass and stored energy (right graph in FIG 4).
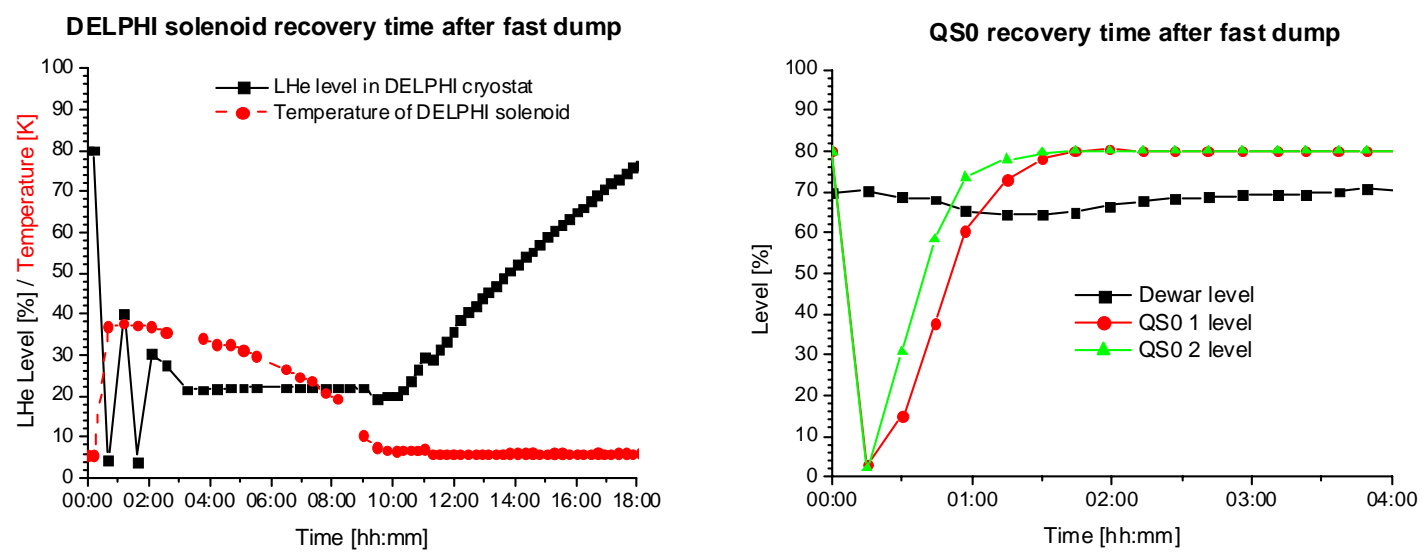

FIGURE 4. Typical solenoid (left) and quadrupole (right) recovery times after a fast energy dump at the DELPHI experiment 

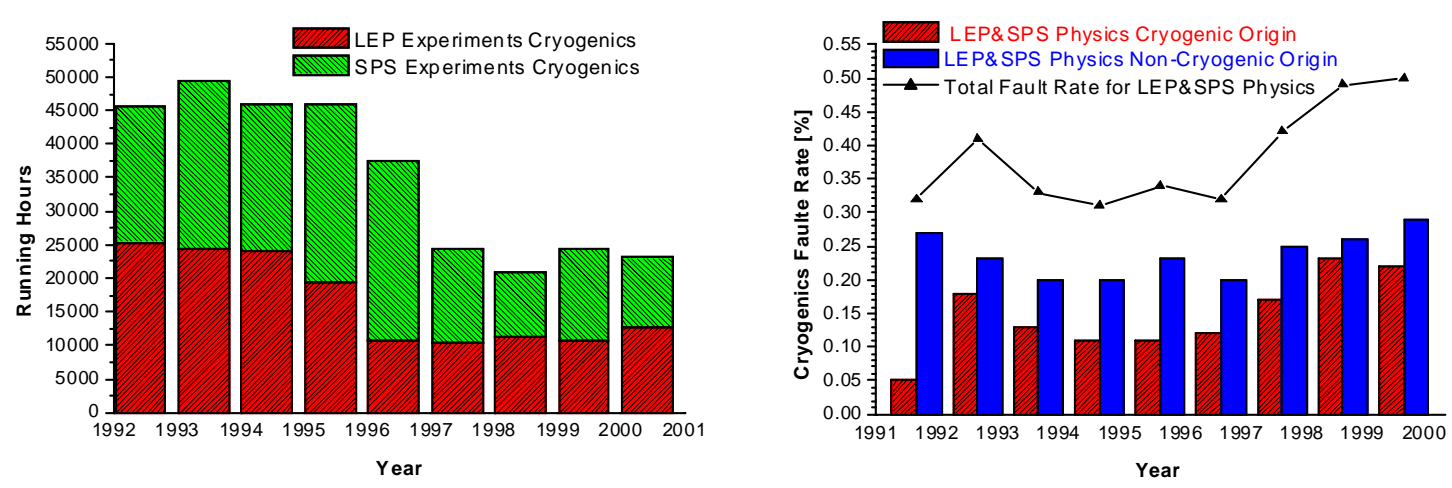

FIGURE 5. Running hours and fault rates of cryogenics for SPS/LEP experiments over the past 9 years [3]

\section{OVERALL OPERATION STATISTICS}

Since 1992 a long-term data acquisition system was established and used to register any cryoplant downtime in a general operation performance statistic. The detailed list showing the main data registered for the cryoplant running for SPS and LEP experiments since 1992 is shown TABLE 3 (see Appendix).

The fault rates are calculated as the ratio of down time and the total number of running hours. They are differentiated between down time caused by failure of cryogenic equipment (cryogenic origin) or provoked by loss of external utilities such as electricity, cooling water and compressed air (non-cryogenic origin).

The total number of running hours accumulated by the cryogenic systems running for the LEP experiments is shown in the first graph of FIG 5 and is compared to the total running time of cryogenics for SPS experiments. In total nearly 150,000 hours were cumulated only by LEP experiment cryogenics. In a first period lasting from 1992 to 1995, four helium refrigerators were running in total around 20,000 hours per year. Two for ALEPH and DELPHI and the other two, each having a cooling power of around $450 \mathrm{~W}$ at 4.5 K, were dedicated to the superconducting quadrupoles at the collision points of the other LEP experiments L3 and OPAL. These plants were removed in 1995 and 1996 to supply SPS experiment cryogenics and their duty was taken over by the main refrigerators for LEP superconducting cavities.

The total 7-years-integral fault rate for the LEP/SPS experiment cryogenics was 5.1 hours down time per 1000 hours of operation time, out of which 2.2 hours were due to problems with cryogenic-origins and 2.9 hours had non-cryogenic origins. For ALEPH and DELPHI cryoplants, the corresponding fault rates were respectively $2.1 \%$ and $3.8 \%$.

\section{CONCLUSION}

During more then 12 years of cryogenics for LEP experiments with very low fault rates we developed a relevant experience concerning operation and maintenance procedures as well as process control and supervisory systems. The ALEPH and DELPHI cryoplants were also part of a pilot project for outsourcing the operation and maintenance, leading to a contract extended to all the CERN cryplants [4]. Furthermore the know-how documented in [5] is being taken into consideration for the design of the cryogenic systems required by the 
next generation physics detectors ATLAS (A Toroidal LHC ApparatuS) and CMS (Compact Muon Solenoid), which will be installed in the collisions points of the new Large Hadron Collider (LHC).

\section{REFERENCES}

1. Dauvergne J.- P., Firth M., Juillerat A.-C., Lebrun P., Rieubland J.-M.; Helium Cryogenics At The LEP Experimental Areas; Advances in Cryogenic Engineering, Vol. 35, P. 901

2. Dauvergne J.- P., Delikaris D., Haug F.; Technical Analysis And Statistics From Long Term Helium Cryoplant Operation with Experimental Superconducting Magnets at CERN; CERN LHC/96-13 (ECR)

3. Barth K. , Dauvergne J.- P., Delikaris D.; LEP1 cryoplants (PA4/PA8), 10th Workshop on LEP-SPS Performance, Ed. by Le Roux P., Poole J. and Truchet M.; CERN-SL-2000-007-DI - pp. 230-232

4. Dauvergene J.-P. and Delikaris D., IT-2719/LHC - OPERATION AND MAINTENANCE OF LIQUID HELIUM CRYOGENIC PLANTS, internal CERN document (Technical Specification)

5. Barth K., Baud R., Dauvergne J.- P. , Delikaris D.; New Long-term Historical Data Recording and Failure Analysis System for the CERN Cryoplants, CERN-LHC-99-005-ECR ; Proceedings of the 20th International Congress of Refrigeration, Vol. 1, Paper 1 
Table 3. Overall statistics for cryogenic operation for Physics Experiments

Cryogenic Operation for Physics Experiments at LEP \& SPS

Statistics from the last seven years operation $(1992,1993,1994,1995,1996,1997,1998,1999)$

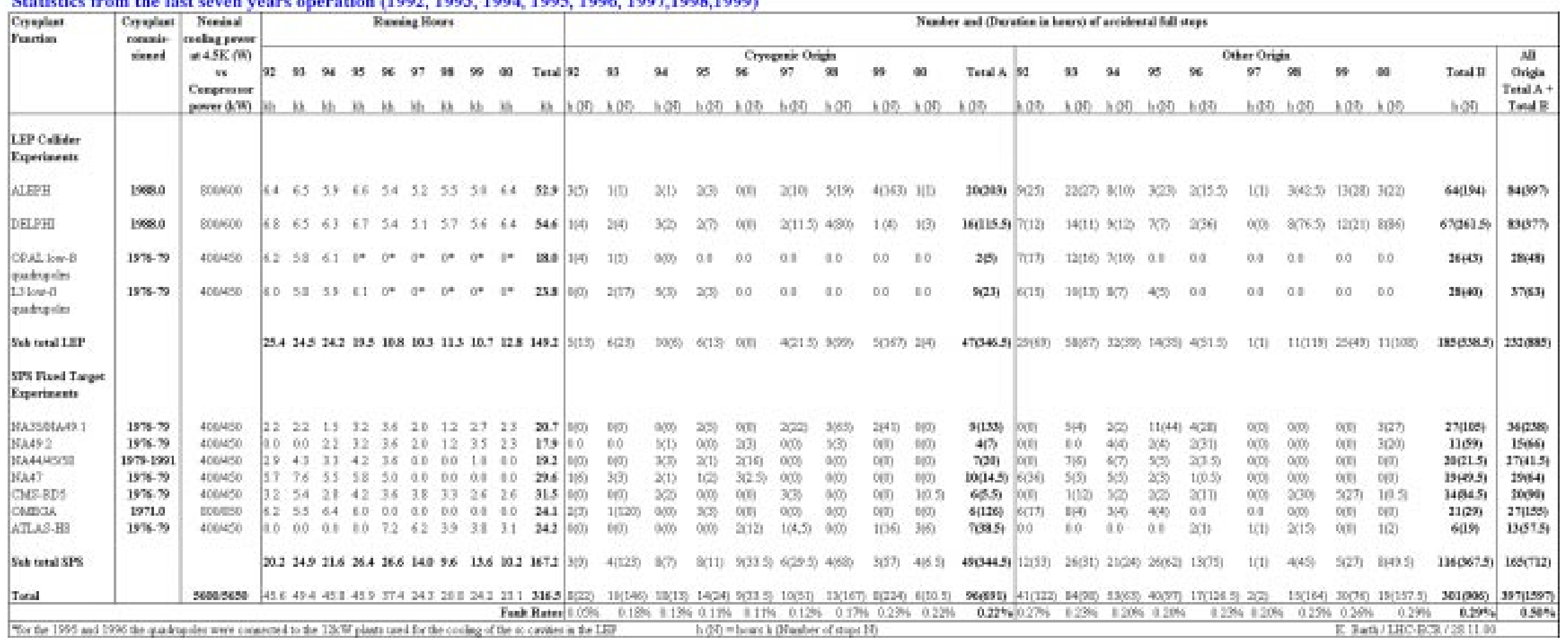

